\title{
ON COMPUTABLE CLASSES OF EQUIDISTANT SETS: EQUIDISTANT FUNCTIONS
}

\author{
CS. VINCZE, A. VARGA, M. OLÁH, AND L. FÓRIÁN \\ Received 11 November, 2016
}

\begin{abstract}
In their paper [6] the authors posed the problem of the characterization of closed subsets in the Euclidean plane that can be realized as the equidistant set of two connected disjoint closed sets. We are going to solve the problem for a special class of equidistant sets. As a main result we give necessary and sufficient conditions for a function to be a so-called equidistant function. This means that its graph is the equidistant set of a line (the first coordinate axis) and the (convex) epigraph of a positive valued convex function satisfying some regularity conditions. In the first step we present a parametric expression for equidistant functions. The parametric expression allows us to use the basic differential geometric tools. We also give a sufficient and necessary condition for a function to be equidistant. In the conluding remarks section some perspectives and extensions of such a special approach will be presented together with some open problems.
\end{abstract}

2010 Mathematics Subject Classification: 51M04

Keywords: generalized conics, equidistant sets

\section{INTRODUCTION: NOTATIONS AND PRELIMINARIES}

Let $K \subset \mathbb{R}^{2}$ be a subset in the Euclidean coordinate plane. The distance between a point $(x, y)$ and $K$ is measured by the usual infimum formula:

$$
d((x, y), K):=\inf \{d((x, y),(a, b)) \mid(a, b) \in K\} .
$$

Let us define the equidistant set of $K$ and $L \subset \mathbb{R}^{2}$ as the set all of whose points have the same distance from $K$ and $L$ :

$$
\{K=L\}:=\left\{(x, y) \in \mathbb{R}^{2} \mid d((x, y), K)=d((x, y), L)\right\} .
$$

The equidistant sets can be considered as a kind of the generalization of conics [6]: $K$ and $L$ are called the focal sets. Equidistant sets are often called midsets too. Their investigations have been started by Wilker's and Loveland's fundamental works [12] and [4]. For another generalization of the classical conics and their applications see

Cs. Vincze was supported by the European Union and the European Social Fund through the project Supercomputer, the national virtual lab (grant no.:TÁMOP-4.2.2.C-11/1/KONV-2012-0010) and by the University of Debrecen's internal research project RH/885/2013. 


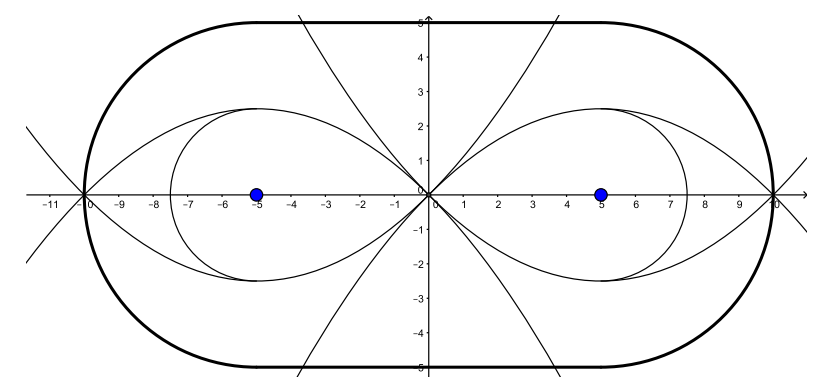

FIGURE 1. The 2D-version of Loveland's example

e.g. [1,5] (polyellipses and their applications), $[2,8,9]$ and [10]. "We find equidistant sets as conventionally defined frontiers in territorial domain controversies: for instance, the United Nations Convention on the Law of the Sea (Article 15) establishes that, in absence of any previous agreement, the delimitation of the territorial sea between countries occurs exactly on the median line every point of which is equidistant of the nearest points to each country"; for the citation see [6]. The points of an equidistant set are difficult to determine in general because there are no simple formulas to compute the distance between a point and a set. On the other hand the equidistant set can have strange and exotic properties even if it has relatively simple focal sets (dimension, connectedness etc.). For some amusing examples see [12] and [4]. Figure 1 shows the 2D-version of Loveland's example in [4]. The equidistant set forms a curve with self-intersection in the middle part of the figure. It consists of two semicircles (left and right hand parts) and four congruent parabolic arcs (the equidistant set of a point and a line). The focal set $K$ contains two different points and $L$ is the boundary of a parallel body ${ }^{1}$ of the segment joining the points in $K$. Therefore the investigation of special classes of equidistant sets seems to be as important as the investigation of the general properties; see e.g. [11], where the authors characterize the equidistant points of finite focal sets in terms of computable constants and parametrization. The process is also implemented in MAPLE. Its motivation is a kind of continuity property of equidistant sets; see Theorem 11 in [6]. Since any compact subset can be approximated by finite subsets with respect to the Hausdorff metric we can approximate the equidistant points of $K$ and $L$ with the equidistant points of finite subsets $K_{n}$ and $L_{n}$; for the details see [11]. In what follows we consider the case of the focal sets

$$
K:=\left\{(t, 0) \in \mathbb{R}^{2} \mid t \in \mathbb{R}\right\} \text { and } L:=\left\{(t, f(t)) \in \mathbb{R}^{2} \mid t \in \mathbb{R}\right\},
$$

where $f: \mathbb{R} \rightarrow \mathbb{R}$ is a positive valued twice continuously differentiable convex function. For the sake of simplicity the equidistant set $\{K=L\}$ will be denoted by $E_{f}$.

\footnotetext{
${ }^{1}$ The parallel body of $L$ with radius $R$ means the union of the closed disks with radius $R$ centered at the points of $L$; see e.g. [3] and [7].
} 
Definition 1. A function $G: \mathbb{R} \rightarrow \mathbb{R}$ is an equidistant function if its graph is the equidistant set of $K$ and $L$ for some positive valued twice continuously differentiable convex function $f$.

In the next section the equidistant funtions are given in terms of a special parametric expression (2.4). Theorem 1 contains the characterization of a parametric expression to be the parametrization of the graph of an equidistant function. Some applications of the basic differential geometric tools (arclength, inclination angle, curvature) are also presented (Subsections 3.1 and 3.2). Theorem 2 contains a simple relationship between the inclination angles of $f$ and its equidistant function at the corresponding positions by the parametric expression. We also give necessary and sufficient conditions for a function $G: \mathbb{R} \rightarrow \mathbb{R}$ to be an equidistant function (Theorem 3). The results give the solution of the problem posed in [6] for a special class of equidistant sets (equidistant functions): characterize all closed sets of the plane that can be realized as the equidistant set of two connected disjoint closed sets. In the conluding remarks section some perspectives and extensions of such a special approach will be presented together with some open problems.

\section{THE PARAMETRIC EXPRESSION OF AN EQUIDISTANT FUNCTION}

Since $f$ is (twice) differentiable, the supporting line of its (convex) epigraph coincides with the tangent line of the function at any $t \in \mathbb{R}$. Using the outer unit normal of the epigraph the equidistant points can be characterized by the formula

$$
(x(t), y(t))=(t, f(t))+y(t) \frac{1}{\sqrt{1+f^{\prime 2}(t)}}\left(f^{\prime}(t),-1\right),
$$

where $y(t)>0$ denotes the (common) distance of the point $(x(t), y(t))$ to the focal sets. Therefore

$$
\begin{gathered}
x(t)=t+\frac{y(t) f^{\prime}(t)}{\sqrt{1+f^{\prime 2}(t)} \text { and } y(t)=f(t)-\frac{y(t)}{\sqrt{1+f^{\prime 2}(t)}}} \\
f(t)=y(t) \cdot\left(1+\frac{1}{\sqrt{1+f^{\prime 2}(t)}}\right)=y(t)\left(\frac{1+\sqrt{1+f^{\prime 2}(t)}}{\sqrt{1+f^{\prime 2}(t)}}\right),
\end{gathered}
$$

i.e.

$$
x(t)=t+\frac{f(t) f^{\prime}(t)}{1+\sqrt{1+f^{\prime 2}(t)}} \text { and } y(t)=\frac{f(t) \sqrt{1+f^{\prime 2}(t)}}{1+\sqrt{1+f^{\prime 2}(t)}} .
$$

\subsection{The geometric interpretation of equidistancy}

Figure 2 shows the case of $f(t)=\frac{1+t^{2}}{3}(t \in \mathbb{R})$ at $t=3$. The equidistant point at $t=3$ (i.e. $x(t)=5$ ) is given as the intersection of the normal line at the tangency point and the bisector of the angle enclosed by the tangent line and the first coordinate axis. 


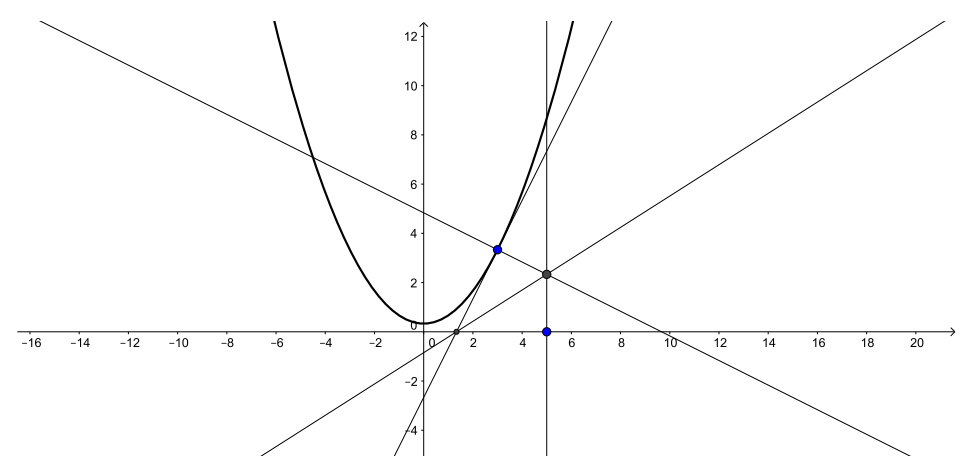

FIGURE 2. Formula (2.1) and Theorem 2

\section{THE CHARACTERIZATION OF THE PARAMETRIC EXPRESSION OF THE} EQUIDISTANT FUNCTIONS

Let

$$
\theta_{f}(t):=\arctan f^{\prime}(t)
$$

be the inclination angle of the function $f$; note that $-\pi / 2<\theta_{f}(t)<\pi / 2$. In terms of the parametrization (2.4)

$$
\begin{aligned}
& \sin \theta_{f}(t)=\frac{f^{\prime}(t)}{\sqrt{1+f^{\prime 2}(t)}}=\frac{x(t)-t}{y(t)} \\
& \cos \theta_{f}(t)=\frac{1}{\sqrt{1+f^{\prime 2}(t)}}=\sqrt{1-\left(\frac{x(t)-t}{y(t)}\right)^{2}}=\frac{\sqrt{y^{2}(t)-(x(t)-t)^{2}}}{y(t)} \\
& \tan \theta_{f}(t)=f^{\prime}(t)=\frac{x(t)-t}{\sqrt{y^{2}(t)-(x(t)-t)^{2}}} .
\end{aligned}
$$

Using the auxiliary function ${ }^{2}$

$$
\varphi(t):=\tan \frac{\theta_{f}(t)}{2}
$$

it follows that

$$
\begin{aligned}
& \sin \theta_{f}(t)=\frac{2 \varphi(t)}{1+\varphi^{2}(t)}, \quad \cos \theta_{f}(t)=\frac{1-\varphi^{2}(t)}{1+\varphi^{2}(t)}, \tan \theta_{f}(t)=\frac{2 \varphi(t)}{1-\varphi^{2}(t)}, \\
& \varphi(t)=\frac{\sin \theta_{f}(t)}{1+\cos \theta_{f}(t)}, \quad \frac{1+\varphi^{2}(t)}{2}=\frac{1}{1+\cos \theta_{f}(t)}
\end{aligned}
$$

\footnotetext{
${ }^{2}$ It is the usual substitution to transform trigonometric expressions into algebraic ones.
} 
note that $-1<\varphi(t)<1$. By (3.1) and (3.3), the parametrization (2.4) can be written as follows:

$$
\begin{aligned}
& x(t)=t+f(t) \frac{\frac{f^{\prime}(t)}{\sqrt{1+f^{\prime 2}(t)}}}{1+\frac{1}{\sqrt{1+f^{\prime 2}(t)}}}=t+f(t) \frac{\sin \theta_{f}(t)}{1+\cos \theta_{f}(t)}=t+f(t) \varphi(t), \\
& y(t)=f(t) \frac{1}{1+\frac{1}{\sqrt{1+f^{\prime 2}(t)}}}=f(t) \frac{1}{1+\cos \theta_{f}(t)}=\frac{1}{2} f(t)\left(1+\varphi^{2}(t)\right) .
\end{aligned}
$$

Excercise 1. Find a geometric argument to conclude (3.4). Hint: see Subsection 2.1 and Figure 2.

Lemma 1. For any $t \in \mathbb{R}: y^{\prime}(t)=x^{\prime}(t) \varphi(t)$.

Proof. Using (3.1) and (3.4)

$$
\begin{aligned}
x^{\prime}(t) & =1+\tan \theta_{f}(t) \varphi(t)+f(t) \varphi^{\prime}(t) \stackrel{(3.3)}{=} 1+\frac{2 \varphi^{2}(t)}{1-\varphi^{2}(t)}+f(t) \varphi^{\prime}(t) \\
& =\frac{1+\varphi^{2}(t)}{1-\varphi^{2}(t)}+f(t) \varphi^{\prime}(t) \\
y^{\prime}(t) & =\frac{1}{2}\left(\tan \theta_{f}(t)\left(1+\varphi^{2}(t)\right)+2 f(t) \varphi(t) \varphi^{\prime}(t)\right) \\
& \stackrel{(3.3)}{=} \frac{\varphi(t)}{1-\varphi^{2}(t)}\left(1+\varphi^{2}(t)\right)+f(t) \varphi(t) \varphi^{\prime}(t)=\varphi(t)\left(\frac{1+\varphi^{2}(t)}{1-\varphi^{2}(t)}+f(t) \varphi^{\prime}(t)\right)
\end{aligned}
$$

as was to be proved.

Theorem 1. Let $x: \mathbb{R} \rightarrow \mathbb{R}$ and $y: \mathbb{R} \rightarrow \mathbb{R}$ be continuously differentiable functions. They give the parametric expression of an equidistant function if and only if

(EF1) for any $t \in \mathbb{R}: y(t)>0$,

(EF2) for any $t \in \mathbb{R}:-1<\frac{x(t)-t}{y(t)}<1$ and the function $t \mapsto \frac{x(t)-t}{y(t)}$ is monotone increasing,

$$
y^{\prime}(t)\left(1+\frac{y(t)}{\sqrt{y^{2}(t)-(x(t)-t)^{2}}}\right)-x^{\prime}(t) \frac{x(t)-t}{\sqrt{y^{2}(t)-(x(t)-t)^{2}}}=0 .
$$

Proof. The necessity of (EF1) is clear. (EF2) follows from (3.1) and the convexity of the function $f$. (EF3) is an equivalent formulation of Lemma 1 :

$$
y^{\prime}(t)-x^{\prime}(t) \varphi(t)=0 \Rightarrow y^{\prime}(t)-x^{\prime}(t) \frac{\sin \theta_{f}(t)}{1+\cos \theta_{f}(t)}=0
$$

because of (3.3). Therefore

$$
y^{\prime}(t)\left(1+\frac{1}{\cos \theta_{f}(t)}\right)-x^{\prime}(t) \tan \theta_{f}(t)=0
$$


and we have the necessity of (EF3) by (3.1). To see the converse statement suppose that (EF1) - (EF3) are satisfied and let us define the function

$$
f(t)=\int_{0}^{t} \frac{x(s)-s}{\sqrt{y^{2}(s)-(x(s)-s)^{2}}} d s+y(0)+\sqrt{y^{2}(0)-x^{2}(0)} .
$$

Using (EF3), a simple calculation shows that the derivative of the function

$$
y(t)+\sqrt{y^{2}(t)-(x(t)-t)^{2}}-f(t)
$$

is zero. By definition (3.8)

$$
y(t)+\sqrt{y^{2}(t)-(x(t)-t)^{2}}-f(t)=y(0)+\sqrt{y^{2}(0)-x^{2}(0)}-f(0)=0
$$

for any $t \in \mathbb{R}$ and, consequently,

$$
y(t)+\sqrt{y^{2}(t)-(x(t)-t)^{2}}=f(t) .
$$

Therefore $f$ is a positively valued function with monotone increasing derivative:

$$
f^{\prime}(t)=\frac{x(t)-t}{\sqrt{y^{2}(t)-(x(t)-t)^{2}}}=\tan \left(\arcsin \frac{x(t)-t}{y(t)}\right)
$$

because of (3.1) and (EF2). Formula (3.11) implies that

$$
\frac{1}{\sqrt{1+f^{\prime 2}(t)}}=\frac{\sqrt{y^{2}(t)-(x(t)-t)^{2}}}{y(t)}
$$

and, by substituting in (3.10) and (3.11), it follows that

$$
y(t)=\frac{f(t) \sqrt{1+f^{\prime 2}(t)}}{1+\sqrt{1+f^{\prime 2}(t)}} \text { and } x(t)=t+\frac{f(t) f^{\prime}(t)}{1+\sqrt{1+f^{\prime 2}(t)}},
$$

i.e. they are just the parametrization of $E_{f}$ in the sense of (2.4).

\subsection{Inclination angle and curvature}

Let $\theta_{e}(t)$ be the inclination angle of the equidistant function at the parameter $t \in \mathbb{R}$. This means that $\theta_{\boldsymbol{e}}(t)$ is the inclination angle belonging to the point $(x(t), y(t))$; it is given by the polar coordinate expression of the tangent vector:

$$
x^{\prime}(t)=v_{e}(t) \cos \theta_{e}(t) \text { and } y^{\prime}(t)=v_{e}(t) \sin \theta_{e}(t),
$$

where $v_{e}(t):=\sqrt{x^{\prime 2}(t)+y^{\prime 2}(t)}$.

Theorem 2. For any $t \in \mathbb{R}: \theta_{e}(t)=\frac{\theta_{f}(t)}{2}$

Proof. By Lemma 1

$$
\begin{aligned}
v_{e}(t) \sin \theta_{e}(t) & =y^{\prime}(t)=x^{\prime}(t) \varphi(t)=v_{e}(t) \cos \theta_{e}(t) \varphi(t) \\
& =v_{e}(t) \cos \theta_{e}(t) \tan \frac{\theta_{f}(t)}{2} .
\end{aligned}
$$


Therefore

$$
\varphi(t)=\tan \frac{\theta_{f}(t)}{2}=\tan \theta_{e}(t) \Rightarrow \theta_{e}(t)=\frac{\theta_{f}(t)}{2}
$$

as was to be proved.

Lemma 2. The parametrization (2.4) is regular because there exists a positive number $\varepsilon$ such that $x^{\prime}(t) \geq \varepsilon>0$ for any $t \in \mathbb{R}$, i.e. $x$ is strictly monotone increasing and its range is the entire real line.

Proof. According to Theorem 2,

$$
-\pi / 4<\theta_{e}(t)<\pi / 4
$$

Since $x^{\prime}(t)=v_{e}(t) \cos \left(\theta_{e}(t)\right)$,

$$
\frac{v_{e}(t)}{\sqrt{2}} \leq x^{\prime}(t) \leq v_{e}(t)
$$

i.e. $x^{\prime}$ is non-negative. If $x^{\prime}(t)=0$ then (EF3) implies that $y^{\prime}(t)=0$. Differentiating

$$
\frac{f^{\prime}(t)}{\sqrt{1+f^{\prime 2}(t)}}=\frac{x(t)-t}{y(t)}
$$

we have

$$
\frac{f^{\prime \prime}(t)\left(\sqrt{1+f^{\prime 2}(t)}-\frac{f^{\prime 2}(t)}{\sqrt{1+f^{\prime 2}(t)}}\right)}{1+f^{\prime 2}(t)}=\frac{\left(x^{\prime}(t)-1\right) y(t)-(x(t)-t) y^{\prime}(t)}{y^{2}(t)}
$$

and, consequently,

$$
\frac{f^{\prime \prime}(t)}{\sqrt{\left(1+f^{\prime 2}(t)\right)^{3}}}=-\frac{1}{y(t)}<0
$$

provided that $x^{\prime}(t)=y^{\prime}(t)=0$. This contradicts to the convexity of $f$. Since $-1<$ $\varphi(t)<1$, the absolute value of $y^{\prime}(t)$ is dominated by $x^{\prime}(t)$ in the sense of Lemma 1. On the other hand formula (3.15) gives a contradiction like (3.16) by a simple continuity argument provided that $x^{\prime}(t)$ is close enough to the zero for some $t \in \mathbb{R}$. Therefore $x^{\prime}(t)$ is uniformly bounded by 0 , i.e. there exists a positive $\varepsilon$ such that $x^{\prime}(t) \geq \varepsilon>0$ for any $t \in \mathbb{R}$ and the range of the function is the entire real line because of the uniform lower bound for the local rate of increasing of the function.

For the computation of the curvature we use the following formulas:

$$
\kappa_{f}(t)=\frac{\theta_{f}^{\prime}(t)}{v_{f}(t)} \text { and } \kappa_{e}(t)=\frac{\theta_{e}^{\prime}(t)}{v_{e}(t)} .
$$

By the previous theorem,

$$
\kappa_{e}(t)=\frac{\theta_{e}^{\prime}(t)}{v_{e}(t)}=\frac{\theta_{f}^{\prime}(t)}{2 v_{e}(t)}=\frac{1}{2} \frac{v_{f}(t)}{v_{e}(t)} \kappa_{f}(t),
$$


where

$$
\begin{aligned}
v_{f}(t) & =\sqrt{1+f^{\prime 2}(t)}=\frac{1}{\cos \theta_{f}(t)}=\frac{1+\varphi^{2}(t)}{1-\varphi^{2}(t)}, \\
\kappa_{f}(t) & =\frac{\theta_{f}^{\prime}(t)}{v_{f}(t)}=2 \frac{(\arctan \varphi)^{\prime}(t)}{v_{f}(t)}=2 \frac{\varphi^{\prime}(t)}{1+\varphi^{2}(t)} \frac{1}{v_{f}(t)} \\
& \stackrel{(3.19)}{=} 2 \varphi^{\prime}(t) \frac{1-\varphi^{2}(t)}{\left(1+\varphi^{2}(t)\right)^{2}}, \\
v_{e}(t) & =\sqrt{x^{\prime 2}(t)+y^{\prime 2}(t)}=\sqrt{x^{\prime 2}(t)\left(1+\varphi^{2}(t)\right)}=x^{\prime}(t) \sqrt{1+\varphi^{2}(t)}
\end{aligned}
$$

because of Lemma 1 and Lemma 2. As formula (3.5) shows

$$
v_{e}(t)=\left(\frac{1+\varphi^{2}(t)}{1-\varphi^{2}(t)}+f(t) \varphi^{\prime}(t)\right) \sqrt{1+\varphi^{2}(t)} .
$$

Therefore

$$
\kappa_{e}(t)=\frac{1}{2} \frac{\frac{1+\varphi^{2}(t)}{1-\varphi^{2}(t)}}{\left(\frac{1+\varphi^{2}(t)}{1-\varphi^{2}(t)}+f(t) \varphi^{\prime}(t)\right) \sqrt{1+\varphi^{2}(t)}} 2 \varphi^{\prime}(t) \frac{1-\varphi^{2}(t)}{\left(1+\varphi^{2}(t)\right)^{2}},
$$

i.e.

$$
\kappa_{e}(t)=\frac{\varphi^{\prime}(t)}{\left(1+\varphi^{2}(t)\right)^{3 / 2}\left(\frac{1+\varphi^{2}(t)}{1-\varphi^{2}(t)}+f(t) \varphi^{\prime}(t)\right)}
$$

Table 1 contains the numerical values of the inclination angle, the magnitude of the velocity and the curvature in case of the function

$$
\begin{gathered}
f(t)=t^{2}+1 \Rightarrow \tan \theta_{f}(t)=f^{\prime}(t)=2 t \\
\varphi(t)=\tan \frac{\arctan (2 t)}{2} \text { and } \varphi^{\prime}(t)=\frac{1}{\cos ^{2} \frac{\arctan (2 t)}{2}} \frac{1}{1+4 t^{2}} .
\end{gathered}
$$

\subsection{An estimation for the arclength between equidistant points}

The arclength between the parameters $a<b$ can be computed as

$$
\mathscr{L}\left(E_{f}\right)_{a}^{b}=\int_{a}^{b} v_{e}(t) d t
$$

note that it is the arclength between the equidistant points $(x(a), y(a))$ and $(x(b), y(b))$. Since $-1<\varphi(t)<1$

$$
\int_{a}^{b} \frac{1+\varphi^{2}(t)}{1-\varphi^{2}(t)}+f(t) \varphi^{\prime}(t) d t \leq \mathscr{L}\left(E_{f}\right)_{a}^{b} \leq \sqrt{2} \int_{a}^{b} \frac{1+\varphi^{2}(t)}{1-\varphi^{2}(t)}+f(t) \varphi^{\prime}(t) d t
$$


ON COMPUTABLE CLASSES OF EQUIDISTANT SETS...

TABLE 1. Some numerical values

\begin{tabular}{|c|c|c|c|c|c|}
\hline & $t=0$ & $t=1$ & $t=2$ & $t=3$ & $t=4$ \\
\hline$x(t)$ & 0 & 2.2361 & 5.9039 & 11.4713 & 19.0073 \\
\hline$y(t)$ & $\frac{1}{2}$ & 1.3820 & 4.0240 & 8.5881 & 15.1241 \\
\hline$\theta_{e}(t)$ & 0 & 0.5536 & 0.6629 & 0.7028 & 0.7232 \\
\hline$v_{e}(t)$ & 2 & 3.2785 & 5.8316 & 8.5804 & 11.3750 \\
\hline$\kappa_{e}(t)$ & $\frac{1}{2}$ & 0.0610 & 0.0101 & 0.0031 & 0.0014 \\
\hline
\end{tabular}

because of (3.22). Here

$$
\begin{aligned}
& \int_{a}^{b} f(t) \varphi^{\prime}(t) d t=f(b) \varphi(b)-f(a) \varphi(a)-\int_{a}^{b} f^{\prime}(t) \varphi(t) d t \\
& \int_{a}^{b} f^{\prime}(t) \varphi(t) d t=\int_{a}^{b} \varphi(t) \tan \theta_{f}(t) d t \stackrel{(3.3)}{=} \int_{a}^{b} \frac{2 \varphi^{2}(t)}{1-\varphi^{2}(t)} d t
\end{aligned}
$$

and, consequently,

$$
b-a+f(b) \varphi(b)-f(a) \varphi(a) \leq \mathscr{L}\left(E_{f}\right)_{a}^{b} \leq \sqrt{2}(b-a+f(b) \varphi(b)-f(a) \varphi(a)) .
$$

Example 1. If $f(t)=1+t^{2}$ then, by using the data of Table 1, we have that

$$
4+17 \tan \frac{0.7232}{2} \approx 10.4299 \leq \mathscr{L}\left(E_{f}\right)_{0}^{4} \leq 14.7501 \approx \sqrt{2}\left(4+17 \tan \frac{0.7232}{2}\right) .
$$

\section{THE CHARACTERIZATION OF THE EQUIDISTANT FUNCTIONS}

In what follows we are looking for the equdistant function in the form

$$
y(t)=G(x(t)), \text { where } G: \mathbb{R} \mapsto \mathbb{R}
$$

Lemma 3. $G$ is a positive valued, twice continuously differentiable convex function. 
Proof. Using Lemma 2, it follows that $G(t)=y \circ x^{-1}(t)$. Therefore

$$
G^{\prime}(t)=y^{\prime}\left(x^{-1}(t)\right) \cdot \frac{1}{x^{\prime}\left(x^{-1}\right)(t)}=\varphi \circ x^{-1}(t)
$$

because of Lemma 1. Since $f$ is twice continuously differentiable and

$$
\varphi=\tan \frac{\arctan f^{\prime}}{2},
$$

we have that $\phi \circ x^{-1}$ is continuously differentiable. The convexity of $G$ follows from the curvature formula (3.18) with $\kappa_{f} \geq 0$ because of the convexity of the function $f$.

Excercise 2. By Lemma 3 we can take the sequence $G_{n}$, where $G_{0}:=f$ and $G_{n+1}: \mathbb{R} \rightarrow \mathbb{R}$ is the equidistant function with respect to $G_{n}$. Prove that $G_{n}(t)$ tends to zero at each $t \in \mathbb{R}$. Hint: see Theorem 2 .

Using that

$$
y^{\prime}(t)=G^{\prime}(x(t)) \cdot x^{\prime}(t)
$$

and $x^{\prime}(t)>0$, condition (EF3) says that

$$
\begin{array}{r}
G^{\prime}(x(t)) \cdot\left(G(x(t))+\sqrt{G^{2}(x(t))-(x(t)-t)^{2}}\right)=x(t)-t, \\
G^{\prime}(x(t)) \cdot \sqrt{G^{2}(x(t))-(x(t)-t)^{2}}=x(t)-t-G^{\prime}(x(t)) \cdot G(x(t)) .
\end{array}
$$

Taking the square of both sides

$$
-G^{2}(x(t)) \cdot(x(t)-t)^{2}=(x(t)-t)^{2}-2(x(t)-t) \cdot G^{\prime}(x(t)) \cdot G(x(t))
$$

We have two possible cases: if $x(t) \neq t$ then

$$
-G^{\prime 2}(x(t)) \cdot(x(t)-t)=(x(t)-t)-2 G^{\prime}(x(t)) \cdot G(x(t)) .
$$

If $x(t)=t$ then, by condition (EF3) in Theorem $1, y^{\prime}(t)=0$. Since

$$
y^{\prime}(t)=G^{\prime}(x(t)) x^{\prime}(t)
$$

we have that $G^{\prime}(x(t))=0$ because of Lemma 2. This means that equation (4.4) holds in this exceptional case too. Therefore

$$
x(t)-t=\frac{2 G^{\prime}(x(t)) \cdot G(x(t))}{1+G^{\prime 2}(x(t))} \Rightarrow x(t)-\frac{2 G^{\prime}(x(t)) \cdot G(x(t))}{1+G^{\prime 2}(x(t))}=t .
$$

Using the auxiliary function

$$
H(x):=x-\frac{2 G^{\prime}(x) \cdot G(x)}{1+G^{\prime 2}(x)}
$$

it follows that $H(x(t))=t$, i.e. $H^{-1}(t)=x(t)$; note that $x^{\prime}(t)>0$ implies the existence of the inverse function. In the sense of Lemma 2 the inverse of $H$ must be of the form $H^{-1}: \mathbb{R} \rightarrow \mathbb{R}$. 
Theorem 3. A twice continuously differentiable function $G: \mathbb{R} \rightarrow \mathbb{R}$ is an equidistant function if and only if

$$
H(x):=x-\frac{2 G^{\prime}(x) \cdot G(x)}{1+G^{\prime 2}(x)}
$$

has a strictly monotone increasing inverse function of the form $H^{-1}: \mathbb{R} \rightarrow \mathbb{R}$ and the parametric expression

$$
x(t):=H^{-1}(t) \text { and } y(t):=G \circ H^{-1}(t)
$$

of $G$ satisfies conditions (EF1), (EF2) and (EF3) of Theorem 1.

Remark 1. The substitution $t=H(x)$ in (EF1) implies that $G$ must be a positive valued function. We also have that $G$ is convex and $-1<G^{\prime}(x)<1$ for any $x \in \mathbb{R}$ because of the monotonicity property (EF2); see the behavior of the function $\frac{2 x}{1+x^{2}}$ on the real line. Since the parametrization in Theorem 3 is based on condition (EF3), it is automatically satisfied: a twice continuously differentiable, positive valued convex function $G: \mathbb{R} \rightarrow \mathbb{R}$ is an equidistant function if and only if

$$
H(x):=x-\frac{2 G^{\prime}(x) \cdot G(x)}{1+G^{\prime 2}(x)}
$$

has a strictly monotone increasing inverse function of the form $H^{-1}: \mathbb{R} \rightarrow \mathbb{R}$ and $-1<G^{\prime}(x)<1$ for any $x \in \mathbb{R}$. Theorem 3 gives the solution of the problem posed in [6] for a special class of equidistant sets (equidistant functions): characterize all closed sets of the plane that can be realized as the equidistant set of two connected disjoint closed sets.

\section{CONCLUDING REMARKS}

\subsection{An open problem}

It can be easily seen that conditions (EF1) - (EF3) are invariant under orientation preserving transformations of the parameter. Can we generalize the characterization of equidistant functions as plane curves in terms of conditions that are invariant under (orientation preserving) Euclidean motions?

\subsection{Generalizations and extensions of the process}

As a simple conclusion of the geometric interpretation of equidistancy (see Subsection 2.1) we have that the equidistant function is the envelope of the family of parabolas such that the first coordinate axis is the common directrix and the focal point is running through the graph of the function $f$. Since the equidistant function takes exactly the form of a parabolic arc in the outer normal cone belonging to any singularity on the graph, the singularities (if they exist at all) do not affect the equidistant function. Moreover, any convex function can be approximated by convex functions of class $\ell^{2}$, i.e. the regularity conditions for the function $f$ can be 
used without loss of generality; see also Theorem 11 in [6] (continuity property of equidistant sets). Another technical simplification is to take the entire real line as the domain of the function $f$. It can be easily seen that if the domain is bounded from above (for example), i.e. $f:(-\infty, b) \rightarrow \mathbb{R}$ then the equidistant function takes the form of an infinite parabolic arc for any $t>\lim _{t \rightarrow b^{-}} x(t)$. We can also take most of unbounded closed convex set in the plane as the epigraph of a convex function by choosing the first coordinate axis parallel to the orthogonal complement of a ray contained in the set. Finally the process presented in the paper gives a local approximation of the equidistant sets in some cases as follows. Consider two disjoint closed convex sets $K$ and $L$ in the plane. For the sake of simplicity we suppose that their boundaries are of class $C^{2}$. If $P$ is an equidistant point of $K$ and $L$ then there exist uniquely determined closest points on $K$ and $L$ to $P: A \in K$ and $B \in L$. The tangent line $k$ of $K$ at the point $A$ provides a linear approximation around $A$ of $K$ containing the closest points of $K$ to the equidistant points around $P$ because of the continuity of the orthogonal projection of the outer points onto the boundary of a closed convex set. If the perpendicular line from $B$ to $k$ intersects the interior of $L$ then we can use the local version of the process to approximate the equidistant set of $K$ and $L$ by an equidistant function around $P$. The previous argument motivates us to use the $\ell^{2}$ boundary in a more effective way by substituting the coordinate axis $k$ with the osculating circle to the boundary of $K$ at the point $A$. This circle provides a higher order contact than the tangent line. Therefore we propose the solution of the problem of the characterization of equidistant sets belonging to the epigraph of a positive valued convex function and the circle given by $x^{2}+(y+R)^{2}=R^{2}$.

\section{REFERENCES}

[1] P. Erdôs and I. Vincze, "On the approximation of closed convex plane curves.” Mat. Lapok, vol. 9, pp. 19-36, 1958.

[2] C. Gross and T.-K. Strempel, "On generalizations of conics and on a generalization of the Fermat-Toricelli problem." Amer. Math. Monthly, vol. 105, no. 8, pp. 732-743, 1998, doi: $10.2307 / 2588990$.

[3] S. R. Lay, Convex Sets and Their Applications. John Wiley and Sons, Inc. New York, 1982.

[4] L. D. Loveland, "When midsets are manifolds." Proc. Amer. Math. Soc., vol. 61, no. 2, pp. 353 360, 1976, doi: 10.1090/S0002-9939-1976-0438342-2.

[5] Z. A. Melzak and J. S. Forsyth, "Polyconics 1. Polyellipses and optimization." Quart. Appl. Math., vol. 35, no. 2, pp. 239-255, 1977, doi: 10.1090/qam/448883.

[6] M. Ponce and S. Santibanez, "On equidistant sets and generalized conics: the old and the new." Amer. Math. Monthly, vol. 121, no. 1, pp. 18-32, 2014, doi: 10.4169/amer.math.monthly.121.01.018.

[7] Cs. Vincze, Convex geometry. University of Debrecen, http://www.tankonyvtar.hu., 2013.

[8] Cs. Vincze and Á. Nagy, "Examples and notes on generalized conics and their applications." AMAPN, vol. 26, pp. 359-357, 2010.

[9] Cs. Vincze and Á. Nagy, "An introduction to the theory of generalized conics and their applications." Journal of Geom. and Phys. , vol. 61, pp. 815-828, 2011, doi: 10.1016/j.geomphys.2010.12.003. 
[10] Cs. Vincze and Á. Nagy, "On the theory of generalized conics with applications in geometric tomography.” J. of Approx. Theory, vol. 164, pp. 371-390, 2012, doi: 10.1016/j.jat.2011.11.004.

[11] Cs. Vincze, A. Varga, M. Oláh, L. Fórián, and S. Lőrinc, " On computable classes of equidistant sets: finite focal sets." accepted for publication in Involve, a journal of Math., 2016.

[12] J. B. Wilker, "Equidistant sets and their connectivity properties." Proc. Amer. Math. Soc., vol. 47, no. 2, pp. 446-452, 1975, doi: 10.2307/2039763.

Authors' addresses

Cs. Vincze

Csaba Vincze, Univ. of Debrecen, Inst. of Math., H-4002 Debrecen, P.O.Box 400, Debrecen, Hungary

E-mail address: csvincze@science.unideb.hu

A. Varga

Adrienn Varga, Univ. of Debrecen, Fac. of Eng., H-4002 Debrecen, P.O.Box 400, Debrecen, Hungary

E-mail address: vargaadeng.unideb.hu

M. Oláh

Márk Oláh, Univ. of Debrecen, BSC mathematics, H-4002 Debrecen, P.O.Box 400, Debrecen, Hungary

E-mail address: olma4000@euromail.hu

L. Fórián

L. Fórián, Univ. of Debrecen, BSC mathematics, H-4002 Debrecen, P.O.Box 400, Debrecen, Hungary

E-mail address: laci.forian@gmail.com 